\title{
Periodic Averaging Principle for Neutral Stochastic Delay Differential Equations with Impulses
}

\author{
Peiguang Wang $(\mathbb{D}$ and Yan Xu $(1)$ \\ College of Mathematics and Information Science, Hebei University, Baoding 071002, Hebei, China \\ Correspondence should be addressed to Yan Xu; xuyanbd@hbu.edu.cn
}

Received 30 March 2020; Revised 9 May 2020; Accepted 29 May 2020; Published 15 June 2020

Guest Editor: Xiaodi Li

Copyright (c) 2020 Peiguang Wang and Yan Xu. This is an open access article distributed under the Creative Commons Attribution License, which permits unrestricted use, distribution, and reproduction in any medium, provided the original work is properly cited.

\begin{abstract}
In this paper, we study the periodic averaging principle for neutral stochastic delay differential equations with impulses under non-Lipschitz condition. By using the linear operator theory, we deal with the difficulty brought by delay term of the neutral system and obtain the conclusion that the solutions of neutral stochastic delay differential equations with impulses converge to the solutions of the corresponding averaged stochastic delay differential equations without impulses in the sense of mean square and in probability. At last, an example is presented to show the validity of the proposed theories.
\end{abstract}

\section{Introduction}

Delay, impulse, and noise are natural phenomena in most practical issues and those phenomena are generally modeled by stochastic delay differential equations with impulses, and noise can be described by Brownian motion. Recent theoretical and computational advancements indicate that stochastic delay differential equations with impulses tend to generate rich and complex dynamics. However, because of the complexity of the system, it is difficult to obtain the exact solution of the vast majority of stochastic delay differential equations with impulses. In this background, it is very important to look for an approximate system which is more amenable for analysis and simulation, and it governs the evolution of the original system over a long time scale.

The averaging principle is an effective method to understand the main part of the behavior of dynamical systems. It allows to avoid the detailed analysis of complex original systems and consider the simplified equations. The first analysis for averaging principle for stochastic differential equations was deeply addressed by Khasminskij [1]. And then the averaging principle has been applied for various types of stochastic differential equations. Generally speaking, the results of averaging principle in standard form mainly fall into two categories. The first one is considered in slow- fast systems or two-time-scale systems. It approximates coupled slow component equation in two-time-scale systems by a noncoupled equation often called an averaged equation. Many important results for averaging principle for two-time-scale stochastic differential equations have been carried out, see, for example, [2-6]. The second is approximating a nonautonomous stochastic differential equation by an autonomous stochastic differential equation. The results obtained by this method can be referred to the papers [7-9] and the references therein. However, there are few results on average principle for neutral stochastic delay differential equations. Recently, averaging principle for stochastic delay differential equations of the neutral type driven by G-Brownian motion was studied [10], in which the impulses are not considered in the system.

Motivated by the previous discussion, in this paper, we study the average principle for the neutral stochastic delay differential equations with impulses. We overcome the difficulties caused by the delay term which is included under the differentiation at the left-hand side, and the impulse appears in neutral stochastic differential equations.

The structure of the paper is the following. In Section 2, we introduce some basic concepts, notations, and necessary hypotheses. In Section 3, under several sufficient conditions, we obtain the main results that the solutions of neutral 
stochastic delay differential equations with impulses converge to the solutions of the corresponding averaged stochastic delay differential equations without impulses in the sense of mean square and in probability. In Section 4, we offer an example to illustrate the effectiveness of the obtained results.

\section{Model Description and Preliminaries}

In this section, we will introduce the basic concepts, the model, and some preliminary lemmas.

Let $\left(\Omega, \mathscr{F},\left\{\mathscr{F}_{t}\right\}_{t \geq 0}, P\right)$ be a complete probability space with a filtration $\left\{\mathscr{F}_{t}\right\}_{t \geq 0}$ satisfying the usual conditions (i.e., it is right continuous and $\mathscr{F}_{0}$ contains all $P$-null sets) and $B(t)$ is $m$-dimensional Brownian motion defined on the space. $\mathscr{K}$ denotes the family of all concave continuous nondecreasing functions $\alpha: \mathbb{R}^{+} \longrightarrow \mathbb{R}^{+}$such that $\alpha(0)=0, \int_{0^{+}}(\mathrm{d} s / \alpha(s))=\infty$. Let $C\left((-\infty, 0] ; \mathbb{R}^{d}\right)$ denote the family of all continuous functions $\xi:(-\infty, 0] \longrightarrow \mathbb{R}^{d}$ with the norm $\|\xi\|=\sup _{-\infty<\theta \leq 0}|\xi(\theta)|$ and $|\cdot|$ denote any norm in $\mathbb{R}^{d}$. Let $C_{\mathscr{F}}^{2}\left((-\infty, 0] ; \mathbb{R}^{d}\right)$ denote the family of all $\mathscr{F}_{t}$-measurable, $C\left((-\infty, 0] ; \mathbb{R}^{d}\right)$-valued random variables $\xi=\left\{\xi(\theta):(-\infty<\theta \leq 0\}\right.$ such that $E\|\xi\|^{2}<\infty$.

In this paper, we will discuss the following neutral stochastic delay differential equations with impulses:

$$
\begin{cases}\mathrm{d}[x(t)+c(t) x(t-\tau)]=f(t, x(t), x(t-\tau)) \mathrm{d} t+g(t, x(t), x(t-\tau)) \mathrm{d} B(t), & t \neq t_{j}, j=1,2, \ldots, l, \\ x\left(t_{j}^{+}\right)-x\left(t_{j}^{-}\right)=I_{j}\left(x\left(t_{j}\right)\right) & t=t_{j}, j=1,2, \ldots, l\end{cases}
$$

where $c \in C((-\infty, U],[0,1))$ is a $T$-periodic function, $0<U<\infty$. The mappings $f:[0, U] \times \mathbb{R}^{d} \times \mathbb{R}^{d} \longrightarrow \mathbb{R}^{d}$ and $g:[0, U] \times \mathbb{R}^{d} \times \mathbb{R}^{d} \longrightarrow \mathbb{R}^{d \times m}$ are Borel measurable and $T$-periodic in the first argument. $I_{j}: \mathbb{R}^{d} \longrightarrow \mathbb{R}^{d}, x\left(t_{j}^{+}\right)$and $x\left(t_{j}^{-}\right)$represent the right and the left limits of $x\left(t_{j}\right), j \in\{1,2, \ldots, l\}$. We assume that there exists a positive constant $l$ such that $t_{j+l}=t_{j}+T, I_{j+l}\left(x\left(t_{j+l}\right)\right)=$ $I_{j}\left(x\left(t_{j}\right)\right), j \in \mathbb{Z}^{+}$, and $[0, T] \cap\left\{t_{j}, j \in \mathbb{Z}^{+}\right\}=\left\{t_{1}, t_{2}, \ldots, t_{l}\right\}$. The initial condition $x_{0}$ is defined by

$$
x_{0}=\xi=\{\xi(t):-\infty<t \leq 0\} \in C_{\mathscr{F}_{t}}^{2}\left((-\infty, 0] ; \mathbb{R}^{d}\right) .
$$
results.

The following lemmas are important to obtain our

Lemma 1 (see [11]). Suppose $H$ is a bounded linear operator on Banach space $X$; if $\|H\|<1$, then $I-H$ has bounded inverse operator $(I-H)^{-1}$ and $\left\|(I-H)^{-1}\right\| \leq(1$ / $(1-\|H\|))$.

Lemma 2 (see [11]). Suppose $H$ is a bounded linear operator on Banach space $X$ and has an inverse bounded operator, for arbitrary $\Delta H: X \longrightarrow X$, if $\|\Delta H\|<\left(1 /\left\|H^{-1}\right\|\right)$, then $S=H+$ $\Delta H$ has a bounded inverse and $S^{-1}=\sum_{j=0}^{\infty}(-1)^{j}$ $H^{-1}\left(H^{-1} \Delta H\right)^{j}$.

Let

$$
\begin{aligned}
P C((-\infty, U]) & =\left\{x:\left.x\right|_{\left(t_{j}, t_{j+1}\right)_{(-\infty, U]}} \in C\left((-\infty, 0] ; \mathbb{R}^{d}\right), x\left(t_{j}^{+}\right)\right. \\
& \left.=x\left(t_{j}\right), j=1,2, \ldots, l\right\},
\end{aligned}
$$

and consider the Banach space

$$
W=\{x: x \in P C((-\infty, U]), x(t)=x(t+T)\} .
$$

Let $\Phi: W \longrightarrow W$ be defined by

$$
(\Phi x)(t)=x(t)+c(t) x(t-\tau)
$$

Lemma 3 (see [12]). Let $c^{M}=\max _{t \in(-\infty, U]} c(t)$. If $0 \leq c(t)<1$, then $\Phi$ has a bounded inverse $\Phi^{-1}$ on $W$, and for all $x \in W$,

$$
\left(\Phi^{-1} x\right)(t)=\sum_{j \geq 0} \prod_{0 \leq i \leq j-1}(-1)^{j} c(t-i \tau) x(t-j \tau)
$$

and $\left\|\Phi^{-1} x\right\| \leq\left(\|x\| /\left(1-c^{M}\right)\right)$.

Since $\Phi$ and $\Phi^{-1}$ are both linear operators, we can change system (1) by using the inverse transformation of $\Phi$ into the following form:

$$
\begin{cases}\mathrm{d} u(t)=f\left(t,\left(\Phi^{-1} u\right)(t),\left(\Phi^{-1} u\right)(t-\tau)\right) \mathrm{d} t+g\left(t,\left(\Phi^{-1} u\right)(t),\left(\Phi^{-1} u\right)(t-\tau)\right) \mathrm{d} B(t), & t \neq t_{j}, j=1,2, \ldots, l, \\ u\left(t_{j}^{+}\right)-u\left(t_{j}^{-}\right)=\left(\Phi I_{j} \Phi^{-1}\right)\left(u\left(t_{j}\right)\right) & t=t_{j}, j=1,2, \ldots, l \\ u(0)=\left(\Phi^{-1} x\right)(0)=u_{0} . & \end{cases}
$$

Hence, $u(t)$ is an T-periodic solution of system (2) if and only if $\left(\Phi^{-1} u\right)(t)$ is an $T$-periodic solution of system (1).
To study the averaging principle of system (1), we impose the following hypotheses on the coefficients. 
$\left(H_{1}\right)$ For all $x_{1}, y_{1}, x_{2}, y_{2} \in \mathbb{R}^{d}$ and $t \in[0, U]$, there exist $\alpha \in \mathscr{K}$ such that

$$
\begin{aligned}
& \left|f\left(t, x_{1}, y_{1}\right)-f\left(t, x_{2}, y_{2}\right)\right|^{2} \vee\left|g\left(t, x_{1}, y_{1}\right)-g\left(t, x_{2}, y_{2}\right)\right|^{2} \\
& \quad \leq \alpha\left(\left|x_{1}-y_{1}\right|^{2}+\left|x_{2}-y_{2}\right|^{2}\right),
\end{aligned}
$$

where $a \vee b:=\max \{a, b\}$
Furthermore, by the definition of $\alpha$, there must exist positive constants $k_{1}$ and $k_{2}$ such that

$$
\alpha(r) \leq k_{1} r+k_{2}, \quad \text { for all } r \geq 0 .
$$

$\left(H_{2}\right)$ For every $x \in \mathbb{R}^{d}$, there exists a positive constant $N_{1}$ such that $\left|I_{j}(x)\right| \leq N_{1}$.

Consider the standard form of system (2)

$$
\begin{cases}\mathrm{d} u_{\epsilon}(t)=\varepsilon f\left(t,\left(\Phi^{-1} u_{\varepsilon}\right)(t),\left(\Phi^{-1} u_{\varepsilon}\right)(t-\tau)\right) \mathrm{d} t+\sqrt{\varepsilon} g\left(t,\left(\Phi^{-1} u_{\varepsilon}\right)(t),\left(\Phi^{-1} u_{\varepsilon}\right)(t-\tau)\right) \mathrm{d} B(t), & t \neq t_{j}, j=1,2, \ldots, l, \\ u_{\varepsilon}\left(t_{j}^{+}\right)-u_{\varepsilon}\left(t_{j}^{-}\right)=\varepsilon\left(\Phi I_{j} \Phi^{-1}\right)\left(u_{\varepsilon}\left(t_{j}\right)\right), & t=t_{j}, j=1,2, \ldots, l, \\ u_{\varepsilon}(0)=u_{0} . & \end{cases}
$$

Accordingly, the standard form of system (1) is

$$
\begin{cases}\mathrm{d}\left[x_{\varepsilon}(t)+c(t) x_{\varepsilon}(t-\tau)\right]=\varepsilon f\left(t, x_{\varepsilon}(t), x_{\varepsilon}(t-\tau)\right) \mathrm{d} t+\sqrt{\varepsilon} g\left(t, x_{\varepsilon}(t), x_{\varepsilon}(t-\tau)\right) \mathrm{d} B(t), & t \neq t_{j}, j=1,2, \ldots, l, \\ x_{\varepsilon}\left(t_{j}^{+}\right)-x_{\varepsilon}\left(t_{j}^{-}\right)=\varepsilon I_{j}\left(x_{\varepsilon}\left(t_{j}\right)\right), & t=t_{j}, j=1,2, \ldots, l, \\ x_{\varepsilon}(0)=x_{0}, & \end{cases}
$$

where the functions $f, g, I_{j}, j \in\{1,2, \ldots, l\}$ have the same conditions as in $\left(H_{1}\right)$ and $\left(H_{2}\right)$, and $\varepsilon \in\left[0, \varepsilon_{0}\right]$ is a positive small parameter with $\varepsilon_{0}$ is a fixed number. Let $\bar{f}(x, y): \mathbb{R}^{d} \times \mathbb{R}^{d} \longrightarrow \mathbb{R}^{d}, \quad \bar{g}(x, y): \mathbb{R}^{d} \times$
$\mathbb{R}^{d} \longrightarrow \mathbb{R}^{d \times m}$, and $\Phi I \Phi^{-1}(x): \mathbb{R}^{d} \longrightarrow \mathbb{R}^{d}$ be measurable functions and satisfy the conditions in $\left(H_{1}\right)-\left(H_{2}\right)$ and the following definitions:

$$
\begin{aligned}
\bar{f}(x, y) & =\frac{1}{T} \int_{0}^{T} f(s, x, y) \mathrm{d} s, \\
\bar{g}(x, y) & =\frac{1}{T} \int_{0}^{T} g(s, x, y) \mathrm{d} s, \\
\overline{\Phi I \Phi^{-1}}(x) & =\frac{1}{T} \sum_{j=1}^{l}\left(\Phi I_{j} \Phi^{-1}\right)(x) .
\end{aligned}
$$

We also assume that the following hypothesis is satisfied.

$\left(H_{3}\right)$ There exists a constant $M>0$ such that $|f(t, x, y)|^{2}<M, \quad|g(t, x, y)|^{2}<M, \quad|\bar{f}(x, y)|^{2}<M, \quad$ and $|\bar{g}(x, y)|^{2}<M$ for every $t \in[0, U]$.

Now, we consider the following averaged stochastic delay differential equations which correspond to the original standard form (10):

$$
\left\{\begin{array}{l}
\mathrm{d} v_{\varepsilon}(t)=\varepsilon\left(\bar{f}\left(\left(\Phi^{-1} v_{\varepsilon}\right)(t),\left(\Phi^{-1} v_{\varepsilon}\right)(t-\tau)\right)+\overline{\Phi I \Phi^{-1}}\left(v_{\varepsilon}(t)\right)\right) \mathrm{d} t \\
+\sqrt{\varepsilon} \bar{g}\left(\left(\Phi^{-1} v_{\varepsilon}\right)(t),\left(\Phi^{-1} v_{\varepsilon}\right)(t-\tau)\right) \mathrm{d} B(t) \\
v_{\varepsilon}(0)=u_{0}
\end{array}\right.
$$

Let $\left(\Phi^{-1} v_{\varepsilon}\right)(t)=y_{\varepsilon}(t)$, for system (11), we consider averaged stochastic delay differential equations:

$$
\left\{\begin{array}{l}
\mathrm{d} y_{\varepsilon}(t)=\varepsilon\left(\bar{f}\left(\left(\Phi^{-1} y_{\varepsilon}\right)(t),\left(\Phi^{-1} y_{\varepsilon}\right)(t-\tau)\right)+\overline{\Phi I \Phi^{-1}}\left(y_{\varepsilon}(t)\right)\right) \mathrm{d} t \\
+\sqrt{\varepsilon} \bar{g}\left(\left(\Phi^{-1} y_{\varepsilon}\right)(t),\left(\Phi^{-1} y_{\varepsilon}\right)(t-\tau)\right) \mathrm{d} B(t) \\
y_{\varepsilon}(0)=x_{0}
\end{array}\right.
$$

Obviously, under hypotheses $\left(H_{1}\right)-\left(H_{2}\right)$, one can follow $[13,14]$ to prove the existence and uniqueness of the periodic probability solutions on $(-\infty, U]$ of the standard form (10) and (11) and the averaged form (13) and (14), respectively. Now, we offer the proof for the relationship between $x_{\varepsilon}(t)$ and $y_{\varepsilon}(t)$.

\section{Main Results and Proofs}

In this section, we will use the periodic averaging principle to investigate the neutral stochastic delay differential equations with impulses. 
In the rest of the paper, $C_{i}$ and $O_{i}, i=1,2,3$, and $P_{j}, j=$ $1,2,3,4$ are all constants. Our main results are the following.

Theorem 1. Suppose hypotheses $\left(H_{1}\right)-\left(H_{3}\right)$ are satisfied and systems (10) and (13)-(11) and (14) have solutions $u_{\varepsilon}, v_{\varepsilon}$, $x_{\varepsilon}$, and $y_{\varepsilon}$, respectively, where $\varepsilon \in\left(0, \varepsilon_{0}\right]$ is a positive small parameter with $\varepsilon_{0}$ a constant, then there exist constants $\varepsilon_{1} \in\left(0, \varepsilon_{0}\right], \quad \mu_{1}>0$, and $\mu_{2}>0$ such that, for any $\varepsilon \in\left(0, \varepsilon_{1}\right], t \in\left[0, \mu_{2} \varepsilon^{-1}\right]$,

$$
E \sup _{t \in\left[0, \mu_{2} \varepsilon^{-1}\right]}\left|u_{\varepsilon}(t)-v_{\varepsilon}(t)\right|^{2} \leq \mu_{1} \varepsilon,
$$

and then, we obtain

$$
E \sup _{t \in\left[0, \mu_{2} \varepsilon^{-1}\right]}\left|x_{\varepsilon}(t)-y_{\varepsilon}(t)\right|^{2} \leq\left\|\Phi^{-1}\right\|^{2} \mu_{1} \varepsilon .
$$

Proof. By using the elementary inequality, for any $t \in[0, U]$, we have

$$
\begin{aligned}
\mid u_{\varepsilon}(t) & -\left.v_{\varepsilon}(t)\right|^{2} \\
\leq & 3 \varepsilon^{2} \mid \int_{0}^{t}\left[f\left(s,\left(\Phi^{-1} u_{\varepsilon}\right)(s),\left(\Phi^{-1} u_{\varepsilon}\right)(s-\tau)\right)\right. \\
& \left.-\bar{f}\left(\left(\Phi^{-1} v_{\varepsilon}\right)(s),\left(\Phi^{-1} v_{\varepsilon}\right)(s-\tau)\right)\right]\left.\mathrm{d} s\right|^{2} \\
& +3 \varepsilon \mid \int_{0}^{t}\left[g\left(s,\left(\Phi^{-1} u_{\varepsilon}\right)(s),\left(\Phi^{-1} u_{\varepsilon}\right)(s-\tau)\right)\right. \\
& \left.-\bar{g}\left(\left(\Phi^{-1} v_{\varepsilon}\right)(s),\left(\Phi^{-1} v_{\varepsilon}\right)(s-\tau)\right)\right]\left.\mathrm{d} B(s)\right|^{2} \\
& +3 \varepsilon^{2}\left|\sum_{j=1}^{\infty}\left(\Phi I_{j} \Phi^{-1}\right)\left(u_{\varepsilon}\left(t_{j}\right)\right)-\int_{0}^{t} \overline{\Phi I \Phi^{-1}}\left(v_{\varepsilon}(t)\right) \mathrm{d} s\right|^{2} \\
:= & J_{1}+J_{2}+J_{3} .
\end{aligned}
$$

For the first term $J_{1}$, thanks again to the elementary inequality yields:

$$
\begin{aligned}
& E \sup _{0 \leq s \leq t} J_{1} \\
& \quad \leq 6 \varepsilon^{2} E \sup _{0 \leq s \leq t}\left|\int_{0}^{s}\left[f\left(\theta,\left(\Phi^{-1} u_{\varepsilon}\right)(\theta),\left(\Phi^{-1} u_{\varepsilon}\right)(\theta-\tau)\right)-f\left(\theta,\left(\Phi^{-1} v_{\varepsilon}\right)(\theta),\left(\Phi^{-1} v_{\varepsilon}\right)(\theta-\tau)\right)\right] \mathrm{d} \theta\right|^{2} \\
& \quad+6 \varepsilon^{2} E \sup _{0 \leq s \leq t}\left|\int_{0}^{s}\left[f\left(\theta,\left(\Phi^{-1} v_{\varepsilon}\right)(\theta),\left(\Phi^{-1} v_{\varepsilon}\right)(\theta-\tau)\right)-\bar{f}\left(\left(\Phi^{-1} v_{\varepsilon}\right)(\theta),\left(\Phi^{-1} v_{\varepsilon}\right)(\theta-\tau)\right)\right] \mathrm{d} \theta\right|^{2}
\end{aligned}
$$

According Hölder's inequality and hypothesis $\left(H_{1}\right)$, we arrive at

$$
\begin{aligned}
& 6 \varepsilon^{2} E \sup _{0 \leq s \leq t}\left|\int_{0}^{s}\left[f\left(\theta,\left(\Phi^{-1} u_{\varepsilon}\right)(\theta),\left(\Phi^{-1} u_{\varepsilon}\right)(\theta-\tau)\right)-f\left(\theta,\left(\Phi^{-1} v_{\varepsilon}\right)(\theta),\left(\Phi^{-1} v_{\varepsilon}\right)(\theta-\tau)\right)\right] \mathrm{d} \theta\right|^{2} \\
& \quad \leq 6 \varepsilon^{2} t k_{1} \int_{0}^{t} E \sup _{0 \leq \theta \leq s}\left[\left|\left(\Phi^{-1} u_{\varepsilon}\right)(\theta)-\left(\Phi^{-1} v_{\varepsilon}\right)(\theta)\right|^{2}+\left|\left(\Phi^{-1} u_{\varepsilon}\right)(\theta-\tau)-\left(\Phi^{-1} v_{\varepsilon}\right)(\theta-\tau)\right|^{2}\right] \mathrm{d} s+6 \varepsilon^{2} t^{2} k_{2} \\
& \quad \leq 6 \varepsilon^{2} t k_{1} \int_{0}^{t}\left\|\Phi^{-1}\right\|^{2} E \sup _{0 \leq \theta \leq s}\left[\left|u_{\varepsilon}(\theta)-v_{\varepsilon}(\theta)\right|^{2}+\left|u_{\varepsilon}(\theta-\tau)-v_{\varepsilon}(\theta-\tau)\right|^{2}\right] \mathrm{d} s+6 \varepsilon^{2} t^{2} k_{2} \\
& \quad \leq 12 \varepsilon^{2} t k_{1}\left\|\Phi^{-1}\right\|^{2} \int_{0}^{t} E \sup _{0 \leq \theta \leq s}\left|u_{\varepsilon}(\theta)-v_{\varepsilon}(\theta)\right|^{2} \mathrm{~d} s+6 \varepsilon^{2} t^{2} k_{2} .
\end{aligned}
$$

Let $n$ be the largest integer such that $n T \leq t$. Then, for every $i=\{1, \ldots, n\}$, we obtain 


$$
\begin{aligned}
& 6 \varepsilon^{2} E \sup _{0 \leq s \leq t}\left|\int_{0}^{s}\left[f\left(\theta,\left(\Phi^{-1} v_{\varepsilon}\right)(\theta),\left(\Phi^{-1} v_{\varepsilon}\right)(\theta-\tau)\right)-\bar{f}\left(\left(\Phi^{-1} v_{\varepsilon}\right)(\theta),\left(\Phi^{-1} v_{\varepsilon}\right)(\theta-\tau)\right)\right] \mathrm{d} \theta\right|^{2} \\
& \leq 12 \varepsilon^{2} E \sup _{0 \leq s \leq t}\left|\sum_{i=1}^{n} \int_{(i-1) T}^{i T}\left[f\left(\theta,\left(\Phi^{-1} v_{\varepsilon}\right)(\theta),\left(\Phi^{-1} v_{\varepsilon}\right)(\theta-\tau)\right)-\bar{f}\left(\left(\Phi^{-1} v_{\varepsilon}\right)(\theta),\left(\Phi^{-1} v_{\varepsilon}\right)(\theta-\tau)\right)\right] \mathrm{d} \theta\right|^{2} \\
& \quad+12 \varepsilon^{2} E \sup _{0 \leq s \leq t}\left|\int_{n T}^{s}\left[f\left(\theta,\left(\Phi^{-1} v_{\varepsilon}\right)(\theta),\left(\Phi^{-1} v_{\varepsilon}\right)(\theta-\tau)\right)-\bar{f}\left(\left(\Phi^{-1} v_{\varepsilon}\right)(\theta),\left(\Phi^{-1} v_{\varepsilon}\right)(\theta-\tau)\right)\right] \mathrm{d} \theta\right|^{2} \\
& \leq 72 \varepsilon^{2} n T \int_{0}^{T} E \sup _{0 \leq \theta \leq s} \alpha\left(\left|\left(\Phi^{-1} v_{\varepsilon}\right)(\theta)-\left(\Phi^{-1} v_{\varepsilon}\right)(i T)\right|^{2}+\left|\left(\Phi^{-1} v_{\varepsilon}\right)(\theta-\tau)-\left(\Phi^{-1} v_{\varepsilon}\right)(i T-\tau)\right|^{2}\right) \mathrm{d} s \\
& \quad+36 \varepsilon^{2} E \sup _{0 \leq s \leq t}\left|\sum_{i=1}^{n} \int_{(i-1) T}^{i T}\left[f\left(\theta,\left(\Phi^{-1} v_{\varepsilon}\right)(i T),\left(\Phi^{-1} v_{\varepsilon}\right)(i T-\tau)\right)-\bar{f}\left(\left(\Phi^{-1} v_{\varepsilon}\right)(i T),\left(\Phi^{-1} v_{\varepsilon}\right)(i T-\tau)\right)\right] \mathrm{d} \theta\right|^{2} \\
& \quad+12 \varepsilon^{2} E \sup _{0 \leq s \leq t}\left|\int_{n T}^{s}\left[f\left(\theta,\left(\Phi^{-1} v_{\varepsilon}\right)(\theta),\left(\Phi^{-1} v_{\varepsilon}\right)(\theta-\tau)\right)-\bar{f}\left(\left(\Phi^{-1} v_{\varepsilon}\right)(\theta),\left(\Phi^{-1} v_{\varepsilon}\right)(\theta-\tau)\right)\right] \mathrm{d} \theta\right|^{2} .
\end{aligned}
$$

By the Hölder's inequality, Burkholder-Davis-Gundy's inequality, and hypotheses $\left(H_{1}\right)$ and $\left(H_{3}\right)$, it follows that

$$
\begin{aligned}
& E \sup _{0 \leq s \leq t}\left|\left(\Phi^{-1} v_{\varepsilon}\right)(s)-\left(\Phi^{-1} v_{\varepsilon}\right)(i T)\right|^{2}+E \sup _{0 \leq s \leq t}\left|\left(\Phi^{-1} v_{\varepsilon}\right)(s-\tau)-\left(\Phi^{-1} v_{\varepsilon}\right)(i T-\tau)\right|^{2} \\
& \leq\left\|\Phi^{-1}\right\|^{2} E \sup _{0 \leq s \leq t}\left|v_{\varepsilon}(i T)-v_{\varepsilon}(i T-\tau)\right|^{2}+2\left\|\Phi^{-1}\right\|^{2} E \sup _{0 \leq s \leq t}\left|v_{\varepsilon}(s)-v_{\varepsilon}(i T)\right|^{2} \\
& \leq C_{1}+C_{2} \varepsilon^{2} E \sup _{0 \leq s \leq t} \int_{i T}^{s}\left|\bar{f}\left(\left(\Phi^{-1} v_{\varepsilon}\right)(\theta),\left(\Phi^{-1} v_{\varepsilon}\right)(\theta-\tau)\right)\right|^{2} \mathrm{~d} \theta \\
& \quad+C_{2} \varepsilon E \sup _{0 \leq s \leq t} \int_{i T}^{s}\left|\bar{g}\left(\left(\Phi^{-1} v_{\varepsilon}\right)(\theta),\left(\Phi^{-1} v_{\varepsilon}\right)(\theta-\tau)\right)\right|^{2} \mathrm{~d} \theta \\
& \leq C_{1}+C_{2} \varepsilon^{2} M T+C_{2} \varepsilon M T, \\
& \quad t \in[(i-1) T, i T] .
\end{aligned}
$$

The definition of $\bar{f}$ implies that

$$
\begin{aligned}
& E \sup _{0 \leq s \leq t}\left|\sum_{i=1}^{n} \int_{(i-1) T}^{i T}\left[f\left(\theta,\left(\Phi^{-1} v_{\varepsilon}\right)(i T),\left(\Phi^{-1} v_{\varepsilon}\right)(i T-\tau)\right)-\bar{f}\left(\left(\Phi^{-1} v_{\varepsilon}\right)(i T),\left(\Phi^{-1} v_{\varepsilon}\right)(i T-\tau)\right)\right] \mathrm{d} \theta\right|^{2} \\
& \quad \leq n \sum_{i=1}^{n} E \sup _{0 \leq s \leq t}\left|\int_{0}^{T} f\left(\theta,\left(\Phi^{-1} v_{\varepsilon}\right)(i T),\left(\Phi^{-1} v_{\varepsilon}\right)(i T-\tau)\right) \mathrm{d} \theta-T \bar{f}\left(\left(\Phi^{-1} v_{\varepsilon}\right)(i T),\left(\Phi^{-1} v_{\varepsilon}\right)(i T-\tau)\right)\right|^{2} \\
& \quad=0 .
\end{aligned}
$$


Thus, we have

$$
\begin{aligned}
E \sup _{0 \leq s \leq t} J_{1} \leq & 12 \varepsilon^{2} t k_{1}\left\|\Phi^{-1}\right\|^{2} \int_{0}^{t} E \sup _{0 \leq \theta \leq s}\left|u_{\varepsilon}(\theta)-v_{\varepsilon}(\theta)\right|^{2} \mathrm{~d} s+6 \varepsilon^{2} t^{2} k_{2} \\
& +72 \varepsilon^{2} n T \int_{0}^{T} E \sup _{0 \leq \theta \leq s}\left[k_{1}\left(\left|\left(\Phi^{-1} v_{\varepsilon}\right)(\theta)-\left(\Phi^{-1} v_{\varepsilon}\right)(i T)\right|^{2}+\left|\left(\Phi^{-1} v_{\varepsilon}\right)(\theta-\tau)-\left(\Phi^{-1} v_{\varepsilon}\right)(i T-\tau)\right|^{2}\right)+k_{2}\right] \mathrm{d} s+48 \varepsilon^{2} t M T \\
\leq & 12 \varepsilon^{2} t k_{1}\left\|\Phi^{-1}\right\|^{2} \int_{0}^{t} E \sup _{0 \leq \theta \leq s}\left|u_{\varepsilon}(\theta)-v_{\varepsilon}(\theta)\right|^{2} \mathrm{~d} s+6 \varepsilon^{2} t^{2} k_{2} \\
& +72 \varepsilon^{2} n k_{1} T^{2}\left(C_{1}+C_{2} \varepsilon^{2} M T+C_{2} \varepsilon M T\right)+72 \varepsilon^{2} n k_{2} T^{2}+48 \varepsilon^{2} t M T \\
:= & \varepsilon O_{1} \int_{0}^{t} E \sup _{0 \leq \theta \leq s}\left|u_{\varepsilon}(\theta)-v_{\varepsilon}(\theta)\right|^{2} \mathrm{~d} s+\varepsilon P_{1} .
\end{aligned}
$$

For the second term $J_{2}$, apply Burkholder-DavisGundy's inequality to deduce

$$
\begin{aligned}
& E \sup _{0 \leq s \leq t} J_{2} \\
& \quad \leq 3 \varepsilon C_{3} E \sup _{0 \leq s \leq t} \int_{0}^{s}\left|g\left(\theta,\left(\Phi^{-1} u_{\varepsilon}\right)(\theta),\left(\Phi^{-1} u_{\varepsilon}\right)(\theta-\tau)\right)-\bar{g}\left(\left(\Phi^{-1} v_{\varepsilon}\right)(\theta),\left(\Phi^{-1} v_{\varepsilon}\right)(\theta-\tau)\right)\right|^{2} \mathrm{~d} \theta \\
& \leq 6 \varepsilon C_{3} E \sup _{0 \leq s \leq t} \int_{0}^{s}\left|g\left(\theta,\left(\Phi^{-1} u_{\varepsilon}\right)(\theta),\left(\Phi^{-1} u_{\varepsilon}\right)(\theta-\tau)\right)-g\left(\theta,\left(\Phi^{-1} v_{\varepsilon}\right)(\theta),\left(\Phi^{-1} v_{\varepsilon}\right)(\theta-\tau)\right)\right|^{2} \mathrm{~d} \theta \\
& \quad+6 \varepsilon C_{3} E \sup _{0 \leq s \leq t} \int_{0}^{s}\left|g\left(\theta,\left(\Phi^{-1} v_{\varepsilon}\right)(\theta),\left(\Phi^{-1} v_{\varepsilon}\right)(\theta-\tau)\right)-\bar{g}\left(\left(\Phi^{-1} v_{\varepsilon}\right)(\theta),\left(\Phi^{-1} v_{\varepsilon}\right)(\theta-\tau)\right)\right|^{2} \mathrm{~d} \theta .
\end{aligned}
$$

Furthermore, on account of hypothesis $\left(H_{1}\right)$, we have

$$
\begin{aligned}
E \sup _{0 \leq s \leq t} \int_{0}^{s}\left|g\left(\theta,\left(\Phi^{-1} v_{\varepsilon}\right)(\theta),\left(\Phi^{-1} v_{\varepsilon}\right)(\theta-\tau)\right)-\bar{g}\left(\left(\Phi^{-1} v_{\varepsilon}\right)(\theta),\left(\Phi^{-1} v_{\varepsilon}\right)(\theta-\tau)\right)\right|^{2} \mathrm{~d} \theta \\
\leq E \sup _{0 \leq s \leq t} \sum_{i=1}^{n} \int_{(i-1) T}^{i T}\left|g\left(\theta,\left(\Phi^{-1} v_{\varepsilon}\right)(\theta),\left(\Phi^{-1} v_{\varepsilon}\right)(\theta-\tau)\right)-\bar{g}\left(\left(\Phi^{-1} v_{\varepsilon}\right)(\theta),\left(\Phi^{-1} v_{\varepsilon}\right)(\theta-\tau)\right)\right|^{2} \mathrm{~d} \theta \\
\quad+E \sup _{0 \leq s \leq t} \int_{n T}^{s}\left|g\left(\theta,\left(\Phi^{-1} v_{\varepsilon}\right)(\theta),\left(\Phi^{-1} v_{\varepsilon}\right)(\theta-\tau)\right)-\bar{g}\left(\left(\Phi^{-1} v_{\varepsilon}\right)(\theta),\left(\Phi^{-1} v_{\varepsilon}\right)(\theta-\tau)\right)\right|^{2} \mathrm{~d} \theta \\
\leq 3 E \sup _{0 \leq s \leq t} \sum_{i=1}^{n} \int_{(i-1) T}^{i T}\left|g\left(\theta,\left(\Phi^{-1} v_{\varepsilon}\right)(\theta),\left(\Phi^{-1} v_{\varepsilon}\right)(\theta-\tau)\right)-g\left(\theta,\left(\Phi^{-1} v_{\varepsilon}\right)(i T),\left(\Phi^{-1} v_{\varepsilon}\right)(i T-\tau)\right)\right|^{2} \mathrm{~d} \theta \\
\quad+3 E \sup _{0 \leq s \leq t} \sum_{i=1}^{n} \int_{(i-1) T}^{i T}\left|g\left(\theta,\left(\Phi^{-1} v_{\varepsilon}\right)(i T),\left(\Phi^{-1} v_{\varepsilon}\right)(i T-\tau)\right)-\bar{g}\left(\left(\Phi^{-1} v_{\varepsilon}\right)(i T),\left(\Phi^{-1} v_{\varepsilon}\right)(i T-\tau)\right)\right|^{2} \mathrm{~d} \theta \\
\quad+3 E \sup _{0 \leq s \leq t} \sum_{i=1}^{n} \int_{(i-1) T}^{i T}\left|\bar{g}\left(\left(\Phi^{-1} v_{\varepsilon}\right)(i T),\left(\Phi^{-1} v_{\varepsilon}\right)(i T-\tau)\right)-\bar{g}\left(\left(\Phi^{-1} v_{\varepsilon}\right)(\theta),\left(\Phi^{-1} v_{\varepsilon}\right)(\theta-\tau)\right)\right|^{2} \mathrm{~d} \theta+4 M T \\
\quad \leq 3 n \int_{0}^{T} \sup _{0 \leq \theta \leq s} \alpha\left(\left|\left(\Phi^{-1} v_{\varepsilon}\right)(\theta)-\left(\Phi^{-1} v_{\varepsilon}\right)(i T)\right|^{2}+\left|\left(\Phi^{-1} v_{\varepsilon}\right)(\theta-\tau)-\left(\Phi^{-1} v_{\varepsilon}\right)(i T-\tau)\right|^{2}\right) \mathrm{d} s \\
\quad+12 n M T+4 M T .
\end{aligned}
$$


Hence, we obtain

$$
\begin{aligned}
E \sup _{0 \leq s \leq t} J_{2} \leq & 12 \varepsilon C_{3} k_{1}\left\|\Phi^{-1}\right\|^{2} \int_{0}^{t} E \sup _{0 \leq \theta \leq s}\left|u_{\varepsilon}(\theta)-v_{\varepsilon}(\theta)\right|^{2} \mathrm{~d} s+6 \varepsilon C_{3} k_{2} t+18 \varepsilon C_{3} n k_{2} T \\
& +18 \varepsilon C_{3} n k_{1} T\left(C_{1}+\varepsilon^{2} C_{2} M T+\varepsilon C_{2} M T\right)+72 \varepsilon C_{3} n M T+24 \varepsilon C_{3} M T \\
:= & \varepsilon O_{2} \int_{0}^{t} E \sup _{0 \leq \theta \leq s}\left|u_{\varepsilon}(\theta)-v_{\varepsilon}(\theta)\right|^{2} \mathrm{~d} s+\varepsilon P_{2} .
\end{aligned}
$$

For the third term $J_{3}$, utilizing hypothesis $\left(H_{2}\right)$, we deduce

$$
\begin{aligned}
E \sup _{0 \leq s \leq t} J_{3} \leq & 6 \varepsilon^{2} l(n+1) E \sup _{0 \leq s \leq t} \sum_{j=1}^{l}\left|\left(\Phi I_{j} \Phi^{-1}\right)\left(u_{\varepsilon}\left(t_{j}\right)\right)\right|^{2} \\
& +6 \varepsilon^{2} \frac{1}{T^{2}} l t E \sup _{0 \leq s \leq t} \sum_{j=1}^{l} \int_{0}^{s}\left|\Phi I_{j} \Phi^{-1}\left(v_{\varepsilon}(\theta)\right)\right|^{2} \mathrm{~d} \theta \\
\leq & 6 \varepsilon^{2} l^{2}(n+1) N_{1}+6 \varepsilon^{2} l^{2}(n+1)^{2} N_{1} \\
:= & \varepsilon P_{3} .
\end{aligned}
$$

Combining (23), (23), and (27), we conclude that

$$
\begin{aligned}
& E \sup _{0 \leq s \leq t}\left|u_{\varepsilon}(s)-v_{\varepsilon}(s)\right|^{2} \\
& \quad \leq \varepsilon\left(O_{1}+O_{2}\right) \int_{0}^{t} E \sup _{0 \leq \theta \leq s}\left|u_{\varepsilon}(\theta)-v_{\varepsilon}(\theta)\right|^{2} \mathrm{~d} s+\varepsilon\left(P_{1}+P_{2}+P_{3}\right) \\
& \quad:=\varepsilon O_{3} \int_{0}^{t} E \sup _{0 \leq \theta \leq s}\left|u_{\varepsilon}(\theta)-v_{\varepsilon}(\theta)\right|^{2} \mathrm{~d} s+\varepsilon P_{4},
\end{aligned}
$$

and in addition, using Gronwall's inequality, we have

$$
E \sup _{0 \leq s \leq t}\left|u_{\varepsilon}(s)-v_{\varepsilon}(s)\right|^{2} \leq \varepsilon P_{4} e^{\varepsilon O_{3} t} .
$$

Therefore, selecting $\mu_{2}>0$ such that, for every $t \in\left[0, \mu_{2} \varepsilon^{-1}\right] \subseteq[0, U]$, setting $\mu_{1}=P_{4} e^{O_{3} \mu_{2}}$, we can choose $\varepsilon_{1} \in\left(0, \varepsilon_{0}\right]$ such that, for each $\varepsilon \in\left(0, \varepsilon_{1}\right]$ and $t \in\left[0, \mu_{2} \varepsilon^{-1}\right]$,

$$
E \sup _{t \in\left[0, \mu_{2} \varepsilon^{-1}\right]}\left|u_{\varepsilon}(t)-v_{\varepsilon}(t)\right|^{2} \leq \mu_{1} \varepsilon,
$$

and then, we obtain

$$
\begin{aligned}
& E \sup _{t \in\left[0, \mu_{2} \varepsilon^{-1}\right]}\left|x_{\varepsilon}(t)-y_{\varepsilon}(t)\right|^{2} \\
& \quad=E \sup _{t \in\left[0, \mu_{2} \varepsilon^{-1}\right]}\left|\left(\Phi^{-1} u_{\varepsilon}\right)(t)-\left(\Phi^{-1} v_{\varepsilon}\right)(t)\right|^{2} \leq\left\|\Phi^{-1}\right\|^{2} \mu_{1} \varepsilon .
\end{aligned}
$$

Corollary 1. Suppose hypotheses $\left(H_{1}\right)-\left(H_{3}\right)$ are satisfied. Then, for any parameter $\gamma>0$, we have

$$
\lim _{\varepsilon \longrightarrow 0} P\left(\left|x_{\varepsilon}(t)-y_{\varepsilon}(t)\right|>\gamma\right)=0 .
$$

Proof. In fact, under the consequence of Theorem 1, by using Chebyshev-Markov inequality, for any given number $\gamma>0$, we can derive

$$
P\left(\left|x_{\varepsilon}(t)-y_{\varepsilon}(t)\right|>\gamma\right) \leq \frac{1}{\gamma^{2}} E\left|x_{\varepsilon}(t)-y_{\varepsilon}(t)\right|^{2} \leq \frac{\left\|\Phi^{-1}\right\|^{2} \mu_{1} \varepsilon}{\gamma^{2}} .
$$

The conclusion follows by letting $\varepsilon \longrightarrow 0$.

The proof is complete.

\section{Illustrative Example}

Example 1. Consider the following neutral stochastic delay differential equations with impulses:

$$
\begin{cases}\mathrm{d}\left[x_{\varepsilon}(t)+\frac{1}{4} x_{\varepsilon}(t-\tau)\right]=\varepsilon\left(x_{\varepsilon}(t)+x_{\varepsilon}(t-\tau)\right) \mathrm{d} t+2 \sqrt{\varepsilon} \cos ^{2} t x_{\varepsilon}(t-\tau) \mathrm{d} B(t), & t \neq t_{j}, j=1,2, \ldots, l, \\ x_{\varepsilon}\left(t_{j}^{+}\right)-x_{\varepsilon}\left(t_{j}^{-}\right)=-0.04 x_{\varepsilon}\left(t_{j}\right), & t=t_{j}, j=1,2, \ldots, l,\end{cases}
$$


where $x_{\varepsilon}(t)=\xi(t), t \in(-\infty, 0]$.

Let $(\Phi x)(t)=x(t)+(1 / 4) x(t-\tau)=u(t)$ and consider the following stochastic delay differential equations with impulses:

$$
\begin{aligned}
& \left\{\begin{array}{l}
\mathrm{d}\left[u_{\varepsilon}(t)\right]=\varepsilon\left(\sum_{i \geq 0}\left(-\frac{1}{4}\right)^{i} u_{\varepsilon}(t-i \tau)+\sum_{i \geq 0}\left(-\frac{1}{4}\right)^{i} u_{\varepsilon}(t-(i+1) \tau)\right) \mathrm{d} t \\
+2 \sqrt{\varepsilon} \cos ^{2} t \sum_{i \geq 0}\left(-\frac{1}{4}\right)^{i} u_{\varepsilon}(t-(i+1) \tau) \mathrm{d} B(t), \quad t \neq t_{j}, j=1,2, \ldots, l, \\
u_{\varepsilon}\left(t_{j}^{+}\right)-u_{\varepsilon}\left(t_{j}^{-}\right)=-0.04 u_{\varepsilon}\left(t_{j}\right), \quad t=t_{j}, j=1,2, \ldots, l,
\end{array}\right. \\
& f\left(t,\left(\Phi^{-1} u_{\varepsilon}\right)(t),\left(\Phi^{-1} u_{\varepsilon}\right)(t-\tau)\right)=\sum_{i \geq 0}\left(-\frac{1}{4}\right)^{i} u_{\varepsilon}(t-i \tau)+\sum_{i \geq 0}\left(-\frac{1}{4}\right)^{i} u_{\varepsilon}(t-(i+1) \tau), \\
& g\left(t,\left(\Phi^{-1} u_{\varepsilon}\right)(t),\left(\Phi^{-1} u_{\varepsilon}\right)(t-\tau)\right)=2 \cos ^{2} t \sum_{i \geq 0}\left(-\frac{1}{4}\right)^{i} u_{\varepsilon}(t-(i+1) \tau), \\
& \left(\Phi I_{j} \Phi^{-1}\right)\left(u_{\varepsilon}\left(t_{j}\right)\right)=-0.04 u_{\varepsilon}\left(t_{j}\right) .
\end{aligned}
$$

Then,

$$
\begin{aligned}
\bar{f}\left(\left(\Phi^{-1} v_{\varepsilon}\right)(t),\left(\Phi^{-1} v_{\varepsilon}\right)(t-\tau)\right) & =\frac{1}{\pi} \int_{0}^{\pi} f\left(t,\left(\Phi^{-1} v_{\varepsilon}\right)(t),\left(\Phi^{-1} v_{\varepsilon}\right)(t-\tau)\right) \mathrm{d} t \\
& =\sum_{i \geq 0}\left(-\frac{1}{4}\right)^{i} v_{\varepsilon}(t-i \tau)+\sum_{i \geq 0}\left(-\frac{1}{4}\right)^{i} v_{\varepsilon}(t-(i+1) \tau), \\
\bar{g}\left(\left(\Phi^{-1} v_{\varepsilon}\right)(t),\left(\Phi^{-1} v_{\varepsilon}\right)(t-\tau)\right) & =\frac{1}{\pi} \int_{0}^{\pi} g\left(t,\left(\Phi^{-1} v_{\varepsilon}\right)(t),\left(\Phi^{-1} v_{\varepsilon}\right)(t-\tau)\right) \mathrm{d} t \\
& =\sum_{i \geq 0}\left(-\frac{1}{4}\right)^{i} v_{\varepsilon}(t-(i+1) \tau), \\
\left.\frac{\Phi I \Phi^{-1}}{\left(v_{\varepsilon}\right.}(t)\right) & =\frac{1}{\pi} \sum_{j=1}^{q}\left(\Phi I_{j} \Phi^{-1}\right)\left(v_{\varepsilon}\left(t_{j}\right)\right),
\end{aligned}
$$

and define a new averaged stochastic delay differential equations:

$$
\begin{aligned}
\mathrm{d} v_{\varepsilon}(t)= & \varepsilon\left(\bar{f}\left(\left(\Phi^{-1} v_{\varepsilon}\right)(t),\left(\Phi^{-1} v_{\varepsilon}\right)(t-\tau)\right)+\overline{\Phi I \Phi^{-1}}\left(v_{\varepsilon}(t)\right)\right) \mathrm{d} t \\
& +\sqrt{\varepsilon} \bar{g}\left(\left(\Phi^{-1} v_{\varepsilon}\right)(t),\left(\Phi^{-1} v_{\varepsilon}\right)(t-\tau)\right) \mathrm{d} B(t),
\end{aligned}
$$


namely,

$$
\begin{aligned}
\mathrm{d} v_{\varepsilon}(t)= & \varepsilon\left(\sum_{i \geq 0}\left(-\frac{1}{4}\right)^{i} v_{\varepsilon}(t-i \tau)+\sum_{i \geq 0}\left(-\frac{1}{4}\right)^{i} v_{\varepsilon}(t-(i+1) \tau)+\frac{1}{\pi} \sum_{j=1}^{q}(-0.04) v_{\varepsilon}\left(t_{j}\right)\right) \mathrm{d} t \\
& +\sqrt{\varepsilon} \sum_{i \geq 0}\left(-\frac{1}{4}\right)^{i} v_{\varepsilon}(t-(i+1) \tau) \mathrm{d} B(t) .
\end{aligned}
$$

Let $\left(\Phi^{-1} v_{\varepsilon}\right)(t)=y_{\varepsilon}(t)$, we have

$$
\begin{aligned}
\mathrm{d} y_{\varepsilon}(t)= & \varepsilon\left(\sum_{i \geq 0}\left(-\frac{1}{4}\right)^{i} y_{\varepsilon}(t-i \tau)+\sum_{i \geq 0}\left(-\frac{1}{4}\right)^{i} y_{\varepsilon}(t-(i+1) \tau)+\frac{1}{\pi} \sum_{j=1}^{q}(-0.04) y_{\varepsilon}\left(t_{j}\right)\right) \mathrm{d} t \\
& +\sqrt{\varepsilon} \sum_{i \geq 0}\left(-\frac{1}{4}\right)^{i} y_{\varepsilon}(t-(i+1) \tau) \mathrm{d} B(t) .
\end{aligned}
$$

When $t \in[0, \tau]$, the solution of system (3) can be deduced as

$$
\begin{aligned}
y_{\varepsilon}(t)= & e^{t}\left\{y_{\varepsilon}(0)+\varepsilon \int_{0}^{t} e^{-s} \sum_{i \geq 1}\left(-\frac{1}{4}\right)^{i} y_{\varepsilon}(s-i \tau) \mathrm{d} s+\varepsilon \int_{0}^{t} e^{-s} \sum_{i \geq 0}\left(-\frac{1}{4}\right)^{i} y_{\varepsilon}(s-(i+1) \tau) \mathrm{d} s\right. \\
& \left.+\varepsilon e^{-t} \frac{1}{\pi} \sum_{j=1}^{q}(-0.04) y_{\varepsilon}\left(t_{j}\right)+\sqrt{\varepsilon} \int_{0}^{t} e^{-s} \sum_{i \geq 0}\left(-\frac{1}{4}\right)^{i} y_{\varepsilon}(s-(i+1) \tau) \mathrm{d} B(s)\right\} .
\end{aligned}
$$

When $t \in[\tau, 2 \tau]$, the solution of system (3) is deduced as

$$
\begin{aligned}
y_{\varepsilon}(t)= & e^{t-\tau}\left\{y_{\varepsilon}(\tau)+\varepsilon \int_{\tau}^{t} e^{-(s-\tau)} \sum_{i \geq 1}\left(-\frac{1}{4}\right)^{i} y_{\varepsilon}(s-i \tau) \mathrm{d} s\right. \\
& +\varepsilon \int_{\tau}^{t} e^{-(s-\tau)} \sum_{i \geq 0}\left(-\frac{1}{4}\right)^{i} y_{\varepsilon}(s-(i+1) \tau) \mathrm{d} s+\varepsilon e^{-(t-\tau)} \frac{1}{\pi} \sum_{j=1}^{q}(-0.04) y_{\varepsilon}\left(t_{j}\right) \\
& \left.+\sqrt{\varepsilon} \int_{\tau}^{t} e^{-(s-\tau)} \sum_{i \geq 0}\left(-\frac{1}{4}\right)^{i} y_{\varepsilon}(s-(i+1) \tau) \mathrm{d} B(s)\right\} .
\end{aligned}
$$

Repeat the steps above on $[2 \tau, 3 \tau],[3 \tau, 4 \tau]$, etc., and we can get the solution $y_{\varepsilon}(t)$ on the whole interval $y_{\varepsilon}(t)$. In addition, it is easy to verify that the hypotheses of Theorem 1 and Corollary 1 holds; hence, the solution of the averaged system (3) converges to that of the standard system (1) in the sense of mean square and in probability.

\section{Conclusion}

In this paper, we generalize the periodic averaging principle for neutral stochastic delay differential equations with impulses. The main difficulties in the application of periodic averaging principle for neutral system are caused by the 
delay term which is included under the differentiation at the left-hand side of the system. Using the linear operator theory, Theorem 1 and Corollary 1 show that the solutions of neutral stochastic delay differential equations with impulses converge to the solutions of the corresponding averaged stochastic delay differential equations without impulses in the sense of mean square and in probability. We remark that when the noises are Le vy processes other than Brownian motion considered in this paper, and the approach presented in the paper remains valid.

\section{Data Availability}

No data were used to support the findings of the study.

\section{Conflicts of Interest}

The authors declare that they have no conflicts of interest.

\section{Authors' Contributions}

All authors completed the paper together. All authors read and approved the final manuscript.

\section{Acknowledgments}

This work was supported by the National Natural Science Foundation of China (NSFC) under Grant nos. 11771115, 11271106, and 11801128 and Natural Science Foundation of Hebei Province under Grant no. A2018201109.

\section{References}

[1] R. Z. Khasminskij, "On the principle of averaging the ito's stochastic differential equations," Kybernetika, vol. 4, no. 3, pp. 260-279, 1968.

[2] S. Cerrai and M. Freidlin, "Averaging principle for a class of stochastic reaction-diffusion equations," Probability Theory and Related Fields, vol. 144, no. 1-2, pp. 137-177, 2009.

[3] Y. Xu, J. Duan, and W. Xu, "An averaging principle for stochastic dynamical systems with Lévy noise," Physica D: Nonlinear Phenomena, vol. 240, no. 17, pp. 1395-1401, 2011.

[4] J. Xu, "L-strong convergence of the averaging principle for slow-fast SPDEs with jumps," Journal of Mathematical Analysis and Applications, vol. 445, no. 1, pp. 342-373, 2017.

[5] P. Wei, Y. Chao, and J. Duan, "Hamiltonian systems with Lévy noise: symplecticity, Hamilton's principle and averaging principle," Physica D: Nonlinear Phenomena, vol. 398, pp. 69-83, 2019.

[6] F. Wu and G. Yin, "An averaging principle for two-time-scale stochastic functional differential equations," Journal of Differential Equations, vol. 269, no. 1, pp. 1037-1077, 2020.

[7] W. Mao, S. You, X. Wu, and X. Mao, "On the averaging principle for stochastic delay differential equations with jumps," Advances in Difference Equations, vol. 2015, no. 1, pp. 1-19, 2015.

[8] Y. Xu, B. Pei, and J.-L. Wu, "Stochastic averaging principle for differential equations with non-lipschitz coefficients driven by fractional brownian motion," Stochastics and Dynamics, vol. 17, no. 2, Article ID 1750013, 2017.
[9] S. Ma and Y. Kang, "Periodic averaging method for impulsive stochastic differential equations with Lévy noise," Applied Mathematics Letters, vol. 93, pp. 91-97, 2019.

[10] X. He, S. Han, and J. Tao, "Averaging principle for sdes of neutral type driven by G-brownian motion," Stochastics and Dynamics, vol. 19, no. 1, Article ID 1950004, 2019.

[11] J. B. Conway, A Course in Functional Analysis, Vol. 96, Springer Science \& Business Media, Berlin, Germany, 2013.

[12] C. Wang, Y. Li, and Y. Fei, "Three positive periodic solutions to nonlinear neutral functional differential equations with impulses and parameters on time scales," Mathematical and Computer Modelling, vol. 52, no. 9-10, pp. 1451-1462, 2010.

[13] M. Ji, W. Qi, Z. Shen, and Y. Yi, "Existence of periodic probability solutions to fokker-planck equations with applications," Journal of Functional Analysis, vol. 277, no. 11, Article ID 108281, 2019.

[14] T. Taniguchi, "Successive approximations to solutions of stochastic differential equations," Journal of Differential Equations, vol. 96, no. 1, pp. 152-169, 1992. 